\title{
ATENDIMENTO DENTARIO DE CRIANÇAS DE OTTO A DOZE ANOS DE IDADE, NOS GRUPOS ESCOLARES ESTADUAIS DA CAPITAL DE SÃO PAULO
}

\begin{abstract}
Souza, J. M. P. de - Atendimento dentário de crianças de oito a doze anos de idade, nos grupos escolares estaduais da Capital de São Paulo. Rev. Saúde públ., S. Paulo, 4:61-70, jun. 1970.

RESUMo - Foram examinadas 915 crianças do sexo masculino, de côr branca, de 8 a 12 anos de idade, com a finalidade de estudar o problema do atendimento dentário na população escolar da Capital do Estado de São Paulo, Brasil, segundo o número de dentistas existentes na escola. O 1.0 molar permanente inferior direito foi utilizado como "indicador de atendimento".
\end{abstract}

\section{1 - INTRODU CXO}

Na Capital do Estado de São Paulo há várias instituições que se propõem dar atendimento dentário a grupos de sua população. Assim, o Serviço Social do Comércio (SESC) mantém consultórios para tratamento de seus associados, a Secretaria da Saúde e Assistência Social do Estado mantém atendimento através de seus postos de saúde, a Prefeitura do Município presta serviços odontológicos nos parques infantis e nos grupos escolares municipais, - Serviço Dentário Escolar da Secretaria da Educação do Estado de São Paulo tem dentistas lotados em vários grupos escolares estaduais, havendo ainda um grande número de órgãos oficiais e particulares de menor tamanho que, de alguma maneira, concorrem para a melhoria da saúde bucal dos habitantes de São Paulo, S.P.
É importante saber-se até que ponto êstes órgãos cumprem suas finalidades. $\mathrm{Pa}$ rece-nos que a tendência é têrmos várias entidades com propósitos iguais ou similares, atuando na mesma área geográfica, cobrindo muitas vêzes a mesma população, geralmente sem um programa bem definido, desconhecendo o que a outra está fazendo, desperdiçando esforços e não conseguindo, aparentemente, atingir seus objetivos.

Uma avaliação da eficácia da ação de tais órgãos deve levar em consideração uma série de fatôres, tais como: população que deveria ser atendida, densidade populacional, nível sócio-econômico, número de dentistas e auxiliares, faixas etárias, níveis de incidência e prevalência da cárie dentária, prioridades, etc.

Recebido para publicação em 27-2-1970.

(1) Do Departamento de Epidemiologia da Faculdade de Saúde Pública da USP - São Paulo, S.P., Brasil. 
SouZA, J. M. P. de - Atendimento dentário de crianças de oito a doze anos de idade, nos grupuí escolares estaduais da Capital de São Paulo. Rev. Saúde públ., S. Paulo, 4:61-70, jun. 1970.

Na Tabela 2 temos a quantidade de crianças examinadas, segundo o tamanho da escola e número de dentistas; 187 meninos $(20,44 \%)$ eram de grupos escolares sem dentistas, $420(45,90 \%)$ de grupos escolares com um dentista e 308 $(33,66 \%)$ de grupos com 2 dentistas.

\section{TABELA 1}

Númemo de grupos escolares segundo dentistas em exercicio e crianças matriculadas.

\begin{tabular}{c|ccc|c}
$\begin{array}{c}\text { N.0 de } \\
\text { dentistas }\end{array}$ & \multicolumn{2}{|c|}{ Crlancas matriculadas } & Total \\
$550-1.200$ & 1.200 & -12.300 & \\
\hline 0 & 5 & 2 & 7 \\
1 & 1 & 8 & 9 \\
2 & 5 & 3 & 8 \\
\hline Total & 11 & 13 & 24 \\
\hline
\end{tabular}

T A B EL A 2

Número de alunos examinados, segundo dentistas em exercicio e criancas matriculadas.

\begin{tabular}{|c|c|c|c|}
\hline $\begin{array}{l}\text { N.० de } \\
\text { dentistas }\end{array}$ & $\begin{array}{c}\text { Criancas } \\
550-1.200\end{array}$ & $\begin{array}{l}\text { matriculadas } \\
1.200-\mid 2.300\end{array}$ & Total \\
\hline 0 & 117 & 70 & 187 \\
\hline 1 & 26 & 394 & 420 \\
\hline 2 & 190 & 118 & 308 \\
\hline Total & 333 & 582 & 915 \\
\hline
\end{tabular}

\section{2. - Métodos}

VIEGAS ${ }^{8,9}$ utiliza o primeiro molar permanente inferior direito -MID. (em con- junto com os incisivos centrais superiores) para estimar o índice CPOD de uma comunidade; observando-se a Figura 2 e a Tabela 6 do seu trabalho ${ }^{9}$, toma-se conhecimento que, aos 7 anos, $66 \%$ dêste dentes já foram atacados por cárie e, aos 8 anos, $82 \%$ (em áreas fluoretadas).

0 primeiro molar inferior direito, da mesma forma que os outros primeiros molares permanentes, precisa merecer atenção total do dentista, pois, até os 9 anos, com a erupção dos segundos pré-molares, os molares são a grande maioria entre os dentes permanentes cariados na bôca, em virtude da baixa prevalência de cárie dentária nos incisivos.

Assim, tomamos o primeiro molar permanente inferior direito como "indicador de atendimento", por considerarmos que um programa bem elaborado deve prever, como uma de suas metas, a restauração (ou, excepcionalmente, a extração) de todos os molares permanentes atacados por cárie. Chamaremos êste dente, indiferentemente, de primeiro molar ou molar per. manente, quando não houver possibilidade de confusão; também usaremos a sigla MID.

No nosso trabalho o MID de cada criança foi examinado e classificado em um dos ítens seguintes:

a) não irrompido;

b) hígido, quando ainda não atacado por cárie;

c) tratado, quando não apresentasse cárie, mas apresentasse restauração permanente ou tivesse sido extraído;

d) necessitando tratamento, quando estivesse cariado.

Consideramos o dente como irrompido quando qualquer parte de sua superficie tivesse rompido a fibromucosa gengival, podendo ser tocada pela ponta da sonda exploradora. Em caso de haver restauração e cárie, o dente era considerado como necessitando tratamento. Os 
SouzA, J. M. P. de - Atendimento dentário de criancas de oito a doze anos de idade, nos grupos escolares estaduais da Capital de São Paulo. Rev. Saúde públ., S. Paulo, 4:61-70, jun. 1970.

critérios de diagnóstico de cárie são os mesmos seguidos em trabalho anterior ${ }^{6}$.

Os exames foram feitos por uma só pessoa (o autor dêste trabalho), usando sonda exploradora número 5 e espêlho plano, com luz natural, na maioria das vêzes na própria sala de aula, estando a criança em pé. Os resultados eram anotados em listas próprias e depois transferidos para fichas IBM; a apuração foi feita utilizando-se a classificadora IBM $82^{(1)}$.

A análise estatística das tabelas de contingência para cada idade, com o fim de verificar a influência do número de dentistas no resultado do atendimento do primeiro molar permanente inferior direito, consistiu na aplicação do método de Goodman ${ }^{4}$, a um nível de significância de $10 \%$, através da estatística $\mathrm{G}^{2}$, com distribuição $\mathrm{X}^{2}$ com 4 graus de liberdade (Tabelas 3 a 7). A Tabela 9 foi analisada pelo mesmo método, modificando-se apenas a quantidade de graus de liberdade para 2.

\section{3 - RESULTADOS E DISCUSSAO}

\section{$3.1-$ Generalidades}

Das 915 crianças examinadas, apenas 6 ainda não tinham o primeiro molar permanente inferior direito erupcionado, sendo 5 com 8 anos e um com 9 anos.

Quanto à porcentagem de primeiros molares atacados, em relação ao total de examinados, segundo as idades, os nossos resultados estão bem próximos daquêles encontrados em Bauru em crianças do sexo masculino ${ }^{(2)}$. Para a devida comparação, damos a seguir as proporçóes de ataque (dentes tratados + dentes necessitando tratamento) encontradas e aquelas de Bauru, com os respectivos números de meninos examinados:

\begin{tabular}{cccccc}
$\begin{array}{c}\text { Idade em } \\
\text { anos }\end{array}$ & 8 & 9 & 10 & 11 & 12 \\
\hline \multirow{2}{*}{ Nossos dados } & $\begin{array}{c}65 \% \\
(198)\end{array}$ & $\begin{array}{c}79 \% \\
(228)\end{array}$ & $\begin{array}{c}85 \% \\
(220)\end{array}$ & $\begin{array}{c}84 \% \\
(172)\end{array}$ & $\begin{array}{c}90 \% \\
(97)\end{array}$ \\
$\begin{array}{c}\text { Dados de } \\
\text { Bauru }\end{array}$ & $\mathbf{7 4 \%}$ & $82 \%$ & $82 \%$ & $79 \%$ & $82 \%$ \\
& $(279)$ & $(304)$ & $(290)$ & $(243)$ & $(174)$
\end{tabular}

\section{2 - Grupo etário de 8 anos}

$\mathrm{O}$ atendimento ao primeiro molar de crianças de 8 anos é analisado na Tabela 3 .

Podemos ver que a ausência de dentista, ou a presença de um ou 2 não alterou pràticamente em nada a necessidade de tratamento, correspondendo respectivamente a $54,84 \%, 52,63 \%, 57,14 \%$ as proporções de primeiros molares que precisam ser atendidos; de fato, êstes resultados não diferem siginificativamente entre si.

Na mesma Tabela observamos que nenhuma das 31 crianças sem dentista apresentou o MID tratado, enquanto que $10,53 \%$ das crianças com um dentista e $13,19 \%$ das com 2 dentistas foram atendidas, sendo ambas as diferenças $(10,53-$ $0 \%$ e $13,19 \%$ - $0 \%$ ) estatìsticamente significantes. Êste fato é importante, pois, aparentemente, a criança de 8 anos só recebe cuidados odontológicos na escola.

\section{3 - Grupo etário de 9 anos}

A idade de 9 anos é analisada a partir da Tabela 4. Neste grupo não encontramos nenhuma diferença significante, havendo apenas uma discreta vantagem para os alunos com 2 dentistas, que tiveram $29,33 \%$ dos MID tratados, contra $14,56 \%$ para um dentista e $16,00 \%$ para nenhum dentista. Isto quer dizer que a quantidade de dentistas nos grupos escolares não influiu no atendimento às crianças.

(1) Do antigo Departamento de Estatística Aplicada d a Faculdade de Saúde Pública da USP.

(2) Dados gentilmente fornecidos pelo Prof. Alfredo Reis Viegas, da Faculdade de Saúde Pública da USP. 
SouzA, J. M. P. de - Atendimento dentário de criancas de oito a doze anos de idade, nos grupos escolares estaduais da Capital de Såo Paulo. Rev. Saúde públ., S. Paulo, 4:61-70, jun. 1970.

\section{TA B E L A 3}

Condição do primeiro molar permanente inferior direito de alunos de 8 anos, segundo dentistas em exercicio.

\begin{tabular}{|c|c|c|c|c|}
\hline Condicãao & \multicolumn{3}{|c|}{ Número de dentistas } & Total \\
\hline Não irrompido ou higido & $\begin{array}{c}14 \\
(45,16 \%)\end{array}$ & $\begin{array}{c}28 \\
(36,84 \%)\end{array}$ & $\begin{array}{c}27 \\
(29,67 \%)\end{array}$ & $\begin{array}{c}69 \\
(34,85 \%)\end{array}$ \\
\hline Tratado & - & $\begin{array}{c}8 \\
(10,53 \%)\end{array}$ & $\begin{array}{c}12 \\
(13,19 \%)\end{array}$ & $\begin{array}{c}20 \\
(10,10 \%)\end{array}$ \\
\hline Necessitando de tratamento & $\begin{array}{c}17 \\
(54,84 \%)\end{array}$ & $\begin{array}{c}40 \\
(52,63 \%)\end{array}$ & $\begin{array}{c}52 \\
(57,14 \%)\end{array}$ & $\begin{array}{c}109 \\
(55,05 \%)\end{array}$ \\
\hline Total & $\begin{array}{c}31 \\
(100,00 \%)\end{array}$ & $\begin{array}{c}76 \\
(100,00 \%)\end{array}$ & $\begin{array}{c}91 \\
(100,00 \%)\end{array}$ & $\begin{array}{c}198 \\
(100,00 \%)\end{array}$ \\
\hline
\end{tabular}

T A B E L A 4

Condição do primeiro molar permanente inferior direito de alunos de 9 anos, segundo dentistas em exercicio.

\begin{tabular}{|c|c|c|c|c|}
\hline \multirow{2}{*}{ Condição } & \multicolumn{3}{|c|}{ Número de dentistas } & \multirow{2}{*}{ Total } \\
\hline & 0 & 1 & 2 & \\
\hline Nâo Irrompido ou higido & $\begin{array}{c}11 \\
(22,00 \%)\end{array}$ & $\begin{array}{c}24 \\
(23,30 \%)\end{array}$ & $\begin{array}{c}12 \\
(16,00 \%)\end{array}$ & $\begin{array}{c}47 \\
(20,61 \%)\end{array}$ \\
\hline Tratado & $\begin{array}{c}8 \\
(16,00 \%)\end{array}$ & $\begin{array}{c}15 \\
(14,56 \%)\end{array}$ & $\begin{array}{c}22 \\
(29,33 \%)\end{array}$ & $\begin{array}{c}45 \\
(19,74 \%)\end{array}$ \\
\hline Necessitando de tratamento & $\begin{array}{c}31 \\
(62,00 \%)\end{array}$ & $\begin{array}{c}64 \\
(62,14 \%)\end{array}$ & $\begin{array}{c}41 \\
(54,67 \%)\end{array}$ & $\begin{array}{c}136 \\
(59,65 \%)\end{array}$ \\
\hline Total & $\begin{array}{c}50 \\
(100,00 \%)\end{array}$ & $\begin{array}{c}103 \\
(100,00 \%)\end{array}$ & $\begin{array}{c}75 \\
(100,00 \%)\end{array}$ & $\begin{array}{c}228 \\
(100,00 \%)\end{array}$ \\
\hline
\end{tabular}

\section{4 - Grupo etário de 10 anos}

Aos 10 anos (Tabela 5), encontramos uma melhor posição para os alunos de escolas com 2 dentistas, embora não significante estatisticamente. Ao compararmos as 33 crianças não atendidas entre as 53 sem assistência dentária escolar $(62,26 \%)$ com os 30 escolares não atendidos entre os 82 com assistência de 2 dentistas (36,59\%), tivemos uma nítida vantagem para êstes últimos; no entanto, quando comparamos sòmente as populaçốes de um profissional com de 2 profissionais, elas se comportam de maneira homogênea, não havendo, neste caso, influência do número de dentistas no atendimento do MID dos alunos.

\section{5 - Grupo etário de 11 anos}

No grupo etário de 11 anos (Tabela 6) observamos o mesmo comportamento en- 
SOUZA, J. M. P. de - Atendimento dentário de crianças de oito a doze anos de idade, nos grupos escolares estaduais da Capital de Săo Paulo. Rev. Saúde públ., S. Paulo, 4:61-70, jun. 1970.

contrado aos 9 anos, ou, qualquer que seja o número de profissionais, não há pràticamente alteração no nível de atendimento.

Chamamos apenas a atenção para o resultado de $0 \%$ em relação a dentes hígidos de alunos que freqüentam escola com 2 dentistas.

Acreditamos que alguns dentes que po- deriam vir a ser considerados como não atacados por cárie já tivessem sido restaurados, o que é perfeitamente compreensível ,pois o clínico algumas vêzes interpreta como atingido um dente que está íntegro para o epidemiologista; é lógico que êste fato deve ter também ocorrido, com maior ou menor intensidade, nos outros grupos etários.

\section{T A B E L A 5}

Condição do primeiro molạr permanente inferior direlto de alunos de 10 anos, segundo dentistas em exercicio.

\begin{tabular}{|c|c|c|c|c|}
\hline Condição & \multicolumn{3}{|c|}{ Número de dentistas } & Total \\
\hline Higido & $\begin{array}{c}4 \\
(7,55 \%)\end{array}$ & $\begin{array}{c}16 \\
(18,82 \%)\end{array}$ & $\begin{array}{c}14 \\
(17,07 \%)\end{array}$ & $\begin{array}{c}34 \\
(15,45 \%)\end{array}$ \\
\hline Tratado & $\begin{array}{c}16 \\
(30,19 \%)\end{array}$ & $\begin{array}{c}31 \\
(36,47 \%)\end{array}$ & $\begin{array}{c}38 \\
(46,34 \%)\end{array}$ & $\begin{array}{c}85 \\
(38,64 \%)\end{array}$ \\
\hline Necessitando de tratamento & $\begin{array}{c}33 \\
(62,26 \%)\end{array}$ & $\begin{array}{c}38 \\
(44,71 \%)\end{array}$ & $\begin{array}{c}30 \\
(36,59 \%)\end{array}$ & $\begin{array}{c}101 \\
(45,91 \%)\end{array}$ \\
\hline Total & $\begin{array}{c}53 \\
(100,00 \%)\end{array}$ & $\begin{array}{c}85 \\
(100,00 \%)\end{array}$ & $\begin{array}{c}82 \\
(100,00 \%)\end{array}$ & $\begin{array}{c}220 \\
(100,00 \%)\end{array}$ \\
\hline
\end{tabular}

TABELA 6

Condiçăo do primeiro molar permanente inferior direito de alunos de 11 anos, segundo dentistas em exercicio.

\begin{tabular}{|c|c|c|c|c|}
\hline \multirow{2}{*}{ Condição } & \multicolumn{3}{|c|}{ Número de dentistas } & \multirow{2}{*}{ Total } \\
\hline & 0 & 1 & 2 & \\
\hline Higido & $\begin{array}{c}6 \\
(18,18 \%)\end{array}$ & $\begin{array}{c}22 \\
(21,15 \%)\end{array}$ & - & $\begin{array}{c}28 \\
(16,28 \%)\end{array}$ \\
\hline Tratado & $\begin{array}{c}12 \\
(36,36 \%)\end{array}$ & $\begin{array}{c}38 \\
(36,54 \%)\end{array}$ & $\begin{array}{c}16 \\
(45,71 \%)\end{array}$ & $\begin{array}{c}66 \\
(38,37 \%)\end{array}$ \\
\hline Necessitando de tratamento & $\begin{array}{c}15 \\
(45,45 \%)\end{array}$ & $\begin{array}{c}44 \\
(42,31 \%)\end{array}$ & $\begin{array}{c}19 \\
(54,29 \%)\end{array}$ & $\begin{array}{c}78 \\
(45,35 \%)\end{array}$ \\
\hline Total & $\begin{array}{c}33 \\
(100,00 \%)\end{array}$ & $\begin{array}{c}104 \\
(100,00 \%)\end{array}$ & $\begin{array}{c}35 \\
(100,00 \%)\end{array}$ & $\begin{array}{c}172 \\
(100,00 \%)\end{array}$ \\
\hline
\end{tabular}


souzA, J. M. P. de - Atendimento dentário de crianças de oito a doze anos de idade, nos grupos escolares estaduais da Capital de São Paulo. Rev. Saüde pübl., S. Paulo, 4:61-70, jun. 1970.

Acreditamos que o critério de prioridade seja um dos mais relevantes, isto porque, em saúde pública, sabemos que as necessidades são sempre maiores que os recursos existentes e os grupos a serem beneficiados por algum serviço devem ser escolhidos segundo alguma escala de va. lôres, a mais lógica e justa possivel.

Segundo um Comitê de Peritos em Hi. giene Dental da Organização Mundial da Saúde ${ }^{5}$, reunido em outubro de 1964, a ordem de prioridade de atendimento dentário pode ser assim estabelecida:

a) crianças em idade escolar primária;

b) crianças em idade escolar secundária ;

c) crianças em idade pré-escolar;

d) doentes crônicos, velhos e indivíduos com defeitos físicos ou mentais;

e) mulheres grávidas;

f) outros grupos.

Acrescenta, ainda, que a preferência deve ser dada aos dentes permanentes, no caso de crianças.

Baseados neste informe técnico, propomo-nos analisar o problema de atendimento dental na população escolar da Capital do Estado de São Paulo, em crianças de 8 a 12 anos de idade, que freqüentam grupos escolares estaduais de mais de 550 até 2.300 alunos matriculados, segundo o número de dentistas que dão êste atendimento.

São nossas hipóteses de trabalho:

a) o número de dentistas em um grupo escolar é proporcional ao número de crianças matriculadas;

b) o nível de tratamento está relacionado com a razão " $n$. . de crianças matriculadas $/ \mathrm{n}^{\circ}$ de dentistas em exercício".

$$
2 \text { - MATERIAL E Metodos }
$$

\section{1 - Material}

Desde agôsto de 1968 vimos conduzindo um pesquisa para determinação da dis. tribuição de probabilidades da variável CPOD. Os dados que utilizamos nêste trabalho são complementares àquela investigação, obtidos a partir daquela época até junho de 1969.

As crianças examinadas pertenciam a 24 grupos escolares sorteados entre grupos da Capital de populaçáo estudantil de mais de 550 a 2.300 alunos, estando representadas nesta amostra tôdas as Delegacias de Ensino, havendo diversificação também quanto aos bairros, tamanho da escola, e número de dentistas.

Em cada grupo escolar anotou-se o número de dentistas, considerando-se, como unidade, um dentista em tempo parcial; assim, um dentista em tempo integral corresponde a 2 dentistas. Não incluímos radiologistas.

Os consultórios dentários encontrados eram dos mais variados tipos, desde uma simples cadeira fixa, até equipamento completo nôvo, com alta rotação.

Foram examinados 915 meninos de côr branca, sendo 198 de 8 anos, 228 de 9 anos, 220 de 10 anos, 172 de 11 anos e 97 de 12 anos, que tivessem nascido e vivido sempre na Capital, a fim de evitar, tanto quanto possível, uma influência nos resultados de uma ação preventiva de fluoretos adicionados à água de abastecimento. $\mathrm{Na}$ data do exame, as crianças poderiam estar numa faixa de idade que abrangia a idade completa menos 45 dias, até a idade completa mais 45 dias.

A Tabela 1 mostra que não havia dentista em 29,17\% dos grupos escolares do estudo, que havia um profissional em $37,50 \%$ e 2 em 33,33\%. Quanto ao número de crianças matriculadas, pràticamente metade das escolas apresentou de 550 a 1.200 crianças. 
SouzA, J. M. P. de - Atendimento dentário de crianças de oito a doze anos de idade, nos grupos escolares estaduais da Capital de São Paulo. Rev. Saúde públ., S. Paulo, 4:61-70, jun. 1970.

\section{6 - Grupo etário de 12 anos}

$\mathrm{Na}$ Tabela 7 , que se refere à idade de 12 anos, verificamos, mais uma vez, que a população de escolares pertencentes a grupos com 2 dentistas tem pràticamente a mesma cobertura que a população com um dentista e a sem dentista.

Resumindo, sòmente nos grupos etários de 8 e 10 anos pudemos notar um atendimento um pouco mais favorável para os primeiros molares inferiores, lado direi- molares atacados por cárie (já tratados + necessitando tratamento) e calculamos as porcentagens de tratamento, segundo a idade e o número de dentistas.

Vemos que as curvas representativas do atendimento de alunos de escolas sem dentistas e com um dentista pràticamente se superpóem. A curva para 2 dentistas, até os 10 anos (onde temos então o valor máximo de atendimento, que passa a ser $55,88 \%$, que consideramos baixo) é levemente superior às outras duas; aos $11 \mathrm{e}$

T A B EL A 7

Condiçăo do primeiro permanente inferior direito de alunos de 12 anos, segundo dentistas em exercicio.

\begin{tabular}{|c|c|c|c|c|}
\hline \multirow{2}{*}{ Condição } & \multicolumn{3}{|c|}{ Número de dentistas } & \multirow{2}{*}{ Total } \\
\hline & 0 & 1 & 2 & \\
\hline Higido & $\begin{array}{c}3 \\
(15,00 \%)\end{array}$ & $\begin{array}{c}\mathbf{5} \\
(9,62 \%)\end{array}$ & $\begin{array}{c}2 \\
(8,00 \%)\end{array}$ & $\begin{array}{c}10 \\
(10,31 \%)\end{array}$ \\
\hline Tratado & $\begin{array}{c}9 \\
(45,00 \%)\end{array}$ & $\begin{array}{c}21 \\
(40,38 \%)\end{array}$ & $\begin{array}{c}10 \\
(40,00 \%)\end{array}$ & $\begin{array}{c}40 \\
(41,24 \%)\end{array}$ \\
\hline Necessitando de tratamento & $\begin{array}{c}8 \\
(40,00 \%)\end{array}$ & $\begin{array}{c}26 \\
(50,00 \%)\end{array}$ & $\begin{array}{c}13 \\
(52,00 \%)\end{array}$ & $\begin{array}{c}47 \\
(48,45 \%)\end{array}$ \\
\hline Total & $\begin{array}{c}20 \\
(100,00 \%)\end{array}$ & $\begin{array}{c}52 \\
(100,00 \%)\end{array}$ & $\begin{array}{c}25 \\
(100,00 \%)\end{array}$ & $\begin{array}{c}97 \\
(100,00 \%)\end{array}$ \\
\hline
\end{tabular}

to, em crianças pertencentes a grupos escolares com 2 dentistas. De qualquer maneira, a porcentagem de atendimento sempre foi muito baixa, nunca alcançando $50 \%$ dos dentes examinados, em qualquer dos grupos. A melhor porcentagem de atendimento foi justamente aos 10 anos, em crianças de grupos escolares com 2 dentistas, cujo nivel foi de $46,34 \%$, correspondendo, também, ao menor nível de necessidade de tratamento, ou seja, $36,59 \%$.

\section{7 - Porcentagem de tratamento}

$\mathrm{Na}$ análise feita até agora, foram tam. bém considerados os dentes hígidos e não irrompidos, fator que poderia ter alguma influência nos resultados. A Figura 1, porém, vem confirmar nossas interpretaçōes. Para sua feitura consideramos apenas os

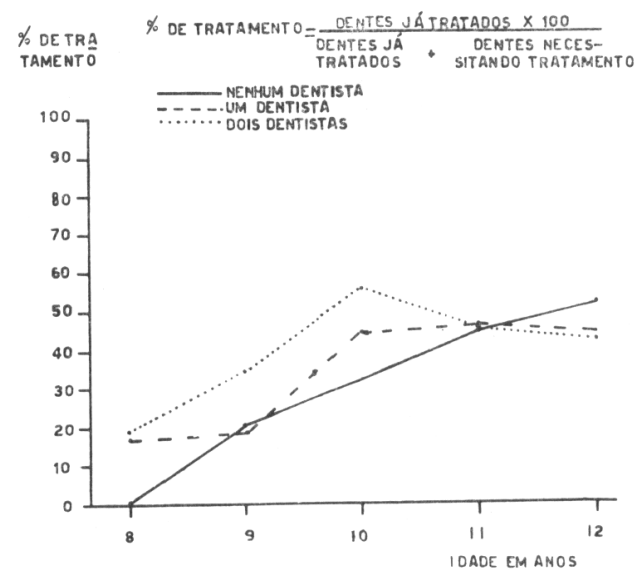

Fig. 1 - Porcentagem de tratamento do primeiro molar permanente inferior direito, segundo idade dos alunos e dentistas em exercício. 
SOUZA, J. M. P. de - Atendimento dentário de crianças de oito a doze anos de idade, nos grupos escolares estaduais da Capital de São Paulo. Rev. Saúde públ., S. Paulo, 4:61-70, Jun. 1970.

12 anos, porém, tôdas as 3 cruvas se equivalem, surpreendendo, além disso, o resultado aos 12 anos, quando a porcentagem de tratamento para meninos sem profissional está em nível superior às porcentagens para um e 2 dentistas, fato que também pode ser observado pela Tabela 7 .

\section{8 - Tipo de tratamento}

Procuramos estudar, também, qual o tipo de trabalho odontológico que o MID apresentava, em função do número de dentistas. As Tabelas 8 e 9 consideram apenas os dentes atendidos e os classifica como extraidos (tratamento radical) ou restaurados (tratamento conservador). Pela Tabela 8, vemos que o atendimento prestado às crianças que não têm dentista na escola é principalmente do tipo radical, não se podendo objetivar uma gradação conforme a idade. Para crianças com um dentista no grupo escolar, ainda temos um alto índice de extrações, mas já se nota uma gradação - nas idades menores, o índice de extração é menor. Para

TA BELA 8

Número de primeiros molares permanentes inferiores, lado direito, tratados segundo idade dos alunos, dentistas em exercício e tipo de tratamento.

\begin{tabular}{|c|c|c|c|c|c|c|c|c|c|c|c|c|}
\hline \multirow{3}{*}{ Idade } & \multicolumn{12}{|c|}{ Número de dentistas } \\
\hline & \multicolumn{5}{|c|}{$\mathbf{0}$} & \multicolumn{4}{|c|}{1} & \multicolumn{3}{|c|}{2} \\
\hline & $\begin{array}{c}\text { Extra- } \\
\text { cões }\end{array}$ & $\begin{array}{c}\text { Res- } \\
\text { taura- } \\
\text { cões }\end{array}$ & Total & $\begin{array}{c}\% \text { de ex- } \\
\text { tracões }\end{array}$ & $\begin{array}{c}\text { Extra- } \\
\text { cões }\end{array}$ & $\begin{array}{c}\text { Res- } \\
\text { taura- } \\
\text { çōes }\end{array}$ & Total & $\begin{array}{l}\% \text { de ex- } \\
\text { trações }\end{array}$ & $\begin{array}{c}\text { Extra- } \\
\text { sões }\end{array}$ & $\begin{array}{l}\text { Res- } \\
\text { taura- } \\
\text { sōes }\end{array}$ & Total & $\begin{array}{c}\% \text { de ex- } \\
\text { trações }\end{array}$ \\
\hline 8 & - & - & - & - & 2 & 6 & 8 & 25,00 & 0 & 12 & 12 & 0,00 \\
\hline 9 & 5 & 3 & 8 & 62,50 & 5 & 10 & 15 & 33,33 & 1 & 21 & 22 & 4,55 \\
\hline 10 & 5 & 11 & 16 & $\mathbf{3 1 , 2 5}$ & 10 & 21 & 31 & 32,26 & 7 & 31 & 38 & 18,42 \\
\hline 11 & 9 & 3 & 12 & 75,00 & 18 & 20 & 38 & 47,37 & 5 & 11 & 16 & 31,25 \\
\hline 12 & 5 & 4 & 9 & $\mathbf{5 5 , 5 6}$ & 13 & 8 & 21 & 61,90 & 4 & 6 & 10 & 40,00 \\
\hline
\end{tabular}

TABELA 9

Total de primeiros molares permanentes inferiores, lado direito, tratados segundo dentistas em exercicio e tipos de tratamento.

\begin{tabular}{|c|c|c|c|c|}
\hline \multirow{2}{*}{ Tratamento } & \multicolumn{3}{|c|}{ Número de dentistas } & \multirow{2}{*}{ Total } \\
\hline & 0 & 1 & 2 & \\
\hline Extraçōes & $\begin{array}{c}24 \\
(53,33 \%)\end{array}$ & $\begin{array}{c}48 \\
(42,48 \%)\end{array}$ & $\begin{array}{c}17 \\
(17,34 \%)\end{array}$ & $\begin{array}{c}89 \\
(34,77 \%)\end{array}$ \\
\hline Restaurações & $\begin{array}{c}21 \\
(46,67 \%)\end{array}$ & $\begin{array}{c}65 \\
(57,52 \%)\end{array}$ & $\begin{array}{c}81 \\
(82,46 \%)\end{array}$ & $\begin{array}{c}167 \\
(65,23 \%)\end{array}$ \\
\hline Total & $\begin{array}{c}45 \\
(100,00 \%)\end{array}$ & $\begin{array}{c}113 \\
(100,00 \%)\end{array}$ & $\begin{array}{c}98 \\
(100,00 \%)\end{array}$ & $\begin{array}{c}256 \\
(100,00 \%)\end{array}$ \\
\hline
\end{tabular}


SOUzA, J. M. P. de - Atendimento dentário de crianças de oito a doze anos de idade, nos grupos escolares estaduais da Capital de São Paulo. Rev. Saúde públ., S. Paulo, 4:61-70, jun. 1970.

a população de escolares com 2 dentistas, abservamos que as porcentagens de extrações são bem menores que as anteriores, havendo, agora, uma escala bem definida segundo as idades. $\mathrm{Na}$ verdade, é o único aspecto que pudemos assinalar como marcantemente positivo em relação aos grupos escolares com 2 dentistas.

Os testes estatísticos a partir da Tabela 9, que considera as idades em conjunto, apresentaram significância quando comparamos a população sujeita a 2 dentistas com a população sujeita a um dentista e quando comparamos os escolares de 2 dentistas com escolares sem dentista; o contraste entre porcentagens de extrações das crianças sem dentista e com um dentista não acusou significância. $\mathrm{Ou}$ seja, quanto ao aspecto de tipo de atendimento, significa pràticamente o mesmo haver um ou nenhum dentista na escola. Quando a escola tem 2 dentistas o tipo de atendimento comparativamente é melhor, sendo mais conservador.

\section{9 - Relação crianças/dentista}

A Tabela 10 nos dá uma síntese da distribuição do total de crianças matriculadas segundo o número de dentistas. Vê-se que, em média, os dentistas que trabalham sòzinhos têm que atender 1.603 crianças, enquanto que cada dentista que trabalha em dupla deve atender 609 escolares, o que nos leva a inferir que o critério de lotação de dentistas não é o número de crianças matriculadas no grupo escolar.

\subsection{0 - Considerações finais}

Devemos observar que, apesar do presente trabalho estar baseado no diagnós- tico de um dente, consideramos os resultados perfeitamente generalizáveis, pois, nas idades estudadas, o primeiro molar permanente inferior direito, bem como qual. quer outro molar, dá uma boa indicação do nível de atendimento dentário, como já expusemos no capítulo de métodos.

Não nos preocupamos, também, em verificar se o atendimento havia sido praticado por profissional da escola ou particular.

Em um programa bem conduzido de atendimento dentário a escolares de nível primário, a cobertura mais importante deve ser conduzida para crianças de 7 e 8 anos, estendendo-se progressivamente para as idades seguintes, segundo um sistema incremental, cujas vantagens não cabem aqui discutir $1,2,3,7$. Dentro dêste espírito, dever-se-ia esperar que as crianças de 8 e 9 anos tivessem suas necessidades de tratamento quase que completamente atendidas, implicando, automàticamente, em níveis elevados de assistência nas idades de 10,11 e 12 anos. Isto porque os dentistas se limitariam a fazer trabalho de revisão em relação aos molares e teriam mais tempo para atender o ataque de cárie a outros dentes.

A partir de nossos resultados, somos levados a acreditar que o atendimento aos escolares não segue esta norma; por motivos que seria interessante estudar, parece que se prefere prestar assistência a crianças na faixa etária de 10 a 12 anos, contrariando-se, assim, uma norma que já provou ser bem sucedida.

De qualquer maneira, chama a atenção a mínima ou nenhuma eficácia da presença quer de um, quer de 2 dentistas,

T A B E L A 10

Total de crianças matriculadas e de dentistas em exercicio nos grupos escolares e número médio de crianças por dentistas, segundo número de dentistas em exercício.

\begin{tabular}{l|crrr}
\hline \multicolumn{1}{c|}{ N.॰ de dentistas } & 0 & 1 & 2 & \multirow{2}{*}{ Total } \\
\hline Crianças matriculadas & 7.242 & 14.425 & 9.739 & 31.406 \\
Dentistas em exercício & - & 9 & 16 & 25 \\
Média: crianças $\div$ dentista & & 1.603 & 609 & 1.256 \\
\hline
\end{tabular}


SOUZA, J. M. P. de - Atendimento dentário de crianças de oito a doze anos de idade, nos grupos escolares estaduais da Capital de São Paulo. Rev. Saúde públ., S. Paulo, 4:61-70, jun. 1970.

pois, como vimos, os resultados obtidos são pràticamente aquêles observados para alunos que não têm profissional na escola. No caso de escolas com sòmente um dentista compreende-se o problema, que é o de ter que atender a um número muito grande de crianças, além de constantemente atender casos de emergência, pro. vocando uma diluição de serviços prestados. Mas, nos grupos escolares servidos por 2 dentistas, o atendimento deveria ser bem superior, haja vista a relação média de 609 alunos para cada dentista.

Observamos que a distribuição dos dentistas nos diversos estabelecimentos de ensino talvez pudesse ser melhorada, usan. do-se critérios objetivos, tais como o de prevalência de necessidades e número de crianças matriculadas.

Vemos, portanto, que não pudemos confirmar nossas hipóteses de trabalho.

$$
4-\text { CONCLUSTES }
$$

1 - Em grupos escolares estaduais da Capital do Estado de São Paulo, o nível de atendimento dentário de crianças de 8 a 12 anos, baseado na condição clínica do primeiro molar permanente inferior direito, é pràticamente o mesmo, quer os alunos pertençam a escola com 2 , um ou nenum dentista.

2 - A presença de 2 dentistas na escola melhora o tipo de assistência dentá. ria para as crianças que são atendidas, pois passa a haver predominância evidente de tratamento conservador, ao invés de radical.

3 - A lotação dos dentistas nos grupos escolares não segue o critério de número de alunos a serem atendidos.

SouzA, J. M. P. de - [Dental care of children aged eight to twelve in public schools of the city of São Paulo, Brazil]. Rev. Saúde públ., S. Paulo, 4:61-70, jun. 1970.
Summary - The problem of dental care in the public schools of the city of São Paulo, Brazil, considering the number of dentists in the school, was studied by examining 915 white children, male, 8 to 12 years old. The permanent first right lower molar was used as "attendance indicator".

\section{5 - REFERÊNCIAS BIBLIOGRAFICAS}

1. CHAVES, M. M. - Odontologia sanitária. Washington, D. C., Organizacion Panamericana de la Salud, 1962 p. 269-81. (OPAS - Public. Cientif., 63).

2. FRANKEL, J. M. \& CHAVES, M. M. Princípios básicos para a organização de um serviço dentário escolar. Rev. bras. Odont., 10:236-48, set./out. 1952.

3. FREIRE, P. S. \& LOURES, O. F. - Organização de um programa dentário escolar em base incremental. Rev. Serv. Saúde públ., Rio de Janeiro, 9:307-25, jul. 1957.

4. GOODMAN, L. A. - Simultaneous confidente intervals for contrasts among multinominal populations. An. math. Statist., 35:716-25, Jun. 1964.

5. ORGANIZACION MUNDIAL DE LA SALUD. Comité de Expertos en Higiene Dental. - Organización de servicios de higiene ciental. Ginebra, 1965. (OMS Ser. Inf. téen., 298).

6. Souza, J. M. P. de et al. - Prevalêneia da cárie dental em brancos e não brancos. Rev. Saúde públ., S. Paulo, 1:38-43, jun. 1967.

7. VIEGAS, A. R. - Aspectos preventivos da cárie dentária. São Paulo, Faculdade de Higiene e Saúde Pública, 1961. p. 7685. (Manual de Odontologia Sanitária, v. 3).

8. VIEGAS, A. R. - Indice simplificado para estimar a prevalência de cárie dental em crianças de 7 a 12 anos de idade. São Paulo, 1968. [Tese para Cátedra - Faculdade de Higiene e Saúde Pública da Univ. São Paulo].

9. VIEGAS, A. R. - Simplified indices for estimating the prevalence of dental carles experience in children seven to twelve years of age. J. publ. Hlth dent., 29: 76-91, Spring 1969. 